\title{
Fixed point theorems for a class of nonlinear sum-type operators and application in a fractional differential equation
}

\author{
Hui Wang ${ }^{1}$, Lingling Zhang ${ }^{1 *}$ and Xiaoqiang Wang ${ }^{2}$
}

\footnotetext{
"Correspondence: tyutzll@163.com ${ }^{1}$ College of Mathematics, Taiyuan University of Technology, Taiyuan, P.R. China

Full list of author information is available at the end of the article
}

\begin{abstract}
In this paper, we consider the fixed point for a class of nonlinear sum-type operators ' $A+B+C$ ' on an ordered Banach space, where $A, B$ are two mixed monotone operators, $C$ is an increasing operator. Without assuming the existence of upper-lower solutions or compactness or continuity conditions, we prove the unique existence of a positive fixed point and also construct two iterative schemes to approximate it. As applications, we research a nonlinear fractional differential equation with multi-point fractional boundary conditions. By using the obtained fixed point theorems of sum-type operator, we get the sufficient conditions which guarantee the existence and uniqueness of positive solutions. At last, a specific example is provided to illustrate our result.
\end{abstract}

MSC: $47 \mathrm{H} 10 ; 47 \mathrm{H} 07 ; 34 \mathrm{~B} 10 ; 34 \mathrm{~B} 18$

Keywords: Sum-type operator; Existence and uniqueness; Positive solution; Fractional differential equation; Fractional boundary condition

\section{Introduction}

With a significant development and extensive applications in various differential and integral equations, nonlinear operators theory has been an active area of research in nonlinear functional analysis. Over the past several decades, much attention has been paid to various fixed point theorems for the single nonlinear operator, and a lot of important results have been obtained, see for example [1-10]. Thereinto, without requiring the operators to be continuous or compact or having the upper-lower solutions, the authors present some important and interesting fixed point theorems (see [1, 4-10]).

On the other hand, in recent years, the sum-type nonlinear operators have been of great interest, there is a great deal of work focused on studying the existence and uniqueness of solutions for sum-type operator equations in a real Banach space, see [11-18]. Consider the following operator equation:

$$
A(x, x)+B x=x .
$$

(c) The Author(s) 2018. This article is distributed under the terms of the Creative Commons Attribution 4.0 International License (http://creativecommons.org/licenses/by/4.0/), which permits unrestricted use, distribution, and reproduction in any medium, provided you give appropriate credit to the original author(s) and the source, provide a link to the Creative Commons license, and indicate if changes were made. 
In [11], the operator $A$ stands for a mixed monotone operator, $B$ represents a sub-linear operator. By using the partial ordering theory and monotone iterative technique, Sang gets the existence and uniqueness of solutions. Note that, in this study, the author does not require the operators to have upper-lower solutions.

In [12], $A: P \times P \rightarrow P$ is a mixed monotone operator verifying a more general concave property, $B: E \times E \rightarrow E$ is a sublinear operator. Amor proves that the operator equation has exactly one fixed point in $\left[u_{0}, v_{0}\right]$.

In [13], $A: P \times P \rightarrow P$ is a mixed monotone operator, $B: P \rightarrow P$ is an increasing subhomogeneous operator or $\alpha$-concave operator. By applying a fixed point theorem for mixed monotone operators, Zhai and Hao get some existence and uniqueness results of positive solutions.

In [14], Zhai and Anderson consider the following operator equation:

$$
A x+B x+C x=x
$$

where $A$ is an increasing $\alpha$-concave operator, $B$ is an increasing sub-homogeneous operator, and $C$ is a homogeneous operator. The authors obtain some new theorems on the existence and uniqueness of positive solutions by using the properties of cones and a fixed point theorem for increasing general $\beta$-concave operator.

In [15], the authors study the fixed point for the sum of two mixed monotone operators.

$$
A(x, x)+B(x, x)=x
$$

where $A, B: P \times P \rightarrow P$ satisfy

$$
A\left(t x, t^{-1} y\right) \geq \psi(t) A(x, y), \quad B\left(t x, t^{-1} y\right) \geq t B(x, y), \quad \psi(t) \in(t, 1], \forall t \in(0,1), x, y \in P .
$$

Various theorems have been established to guarantee the existence of a unique positive solution. In addition, associated iterative schemes have been established for finding the approximate solution converging to the obtained fixed point.

In [16], the authors research the existence and uniqueness of positive solution to the following sum-type mixed monotone operator equation:

$$
A(x, x)+B(x, x)+C(x, x)=x
$$

where $A, B, C: P \times P \rightarrow P$ are three general mixed monotone operators, $A, B$ satisfy the same condition in [15], and $C(\cdot, y): P \rightarrow P$ is concave for fixed $y ; C(x, \cdot): P \rightarrow P$ is convex for any fixed $x$. The authors establish various theorems to guarantee the existence of unique positive solution and they also construct iterative schemes to approximate the unique solution.

In [17], we have already studied the following sum-type operator equation:

$$
A x+B x+C(x, x)=x,
$$

where $A: P \rightarrow P$ is an increasing $\alpha$-concave (or sub-homogeneous) operator, $B: P \rightarrow P$ is a decreasing operator, $C: P \times P \rightarrow P$ is a mixed monotone operator. By using the properties 
of cones and a fixed point theorem for mixed monotone operator, we obtain the existence and uniqueness of positive solutions.

As applications, the above results on the sum-type nonlinear operators have been widely applied to study nonlinear differential and integral equations, see [11-23] and the references therein. In [12], the new fixed point theorems are used to prove positive solutions to a second order Neumann boundary value problem, a Sturm-Liouville boundary value problem, and a nonlinear elliptic boundary value problem for the Lane-Emden-Fowler equation. In $[13,19,20]$, authors investigate the existence and uniqueness results for some kinds of fractional differential equations and nonlinear elastic beam equations via the fixed point theorems in [13]. Also, based on a method originally due to Zhai and Anderson [14], Feng et al. present the existence and uniqueness of positive solutions for nonlinear elastic beam equations, Lane-Emden-Fowler equations, and a class of fractional differential equation with integral boundary conditions in [14,21]. Besides, these fixed point theorems can also be used to deal with some singular problems. In [22, 23], the unique existence of positive solutions for singular fractional differential systems with coupled integral boundary conditions and with integral boundary conditions is studied by fixed point theorem in [15].

Motivated by the above work on fixed point theorems of sum-type operator and their wide applications, in this paper, we continue to talk bout another sum-type operator equation on an ordered Banach space.

$$
A(x, x)+B(x, x)+C x=x
$$

where $A, B: P \times P \rightarrow P$ are two mixed monotone operators, $C: P \rightarrow P$ is an increasing operator. We are concerned with the existence and uniqueness of positive solutions for this kind of operator equation, in which the operators are not required to be continuous, compact but satisfy the following properties.

Case one:

(1) $A\left(t x, t^{-1} y\right) \geq t^{\alpha} A(x, y), \alpha \in(0,1), \forall x, y \in P, t \in(0,1)$.

(2) $B(\cdot, y): P \rightarrow P$ is concave for fixed $y ; B(x, \cdot): P \rightarrow P$ is convex for any fixed $x$.

(3) $C$ is a sub-homogeneous operator.

Case two:

(1) $A\left(t x, t^{-1} y\right) \geq t A(x, y), \forall x, y \in P, t \in(0,1)$.

(2) $B(\cdot, y): P \rightarrow P$ is concave for fixed $y ; B(x, \cdot): P \rightarrow P$ is convex for any fixed $x$.

(3) $C$ is a $\alpha$-concave operator.

After obtaining the unique existence results for operator Eq. (1.1), we also construct two iterative sequences for uniformly approximating the positive solution. Then, we utilize the obtained fixed point theorems to study the existence and uniqueness of positive solutions for a nonlinear fractional differential equation with multi-point fractional boundary conditions. Also, we give a specific example to demonstrate our abstract result. The characteristic features presented in this paper are as follows. Firstly, to our knowledge, in the existing literature, there is almost no research on the fixed point for sum-type operator ' $A+B+C^{\prime}$ with operators satisfying the conditions showed in case one and case two. Hence, our research presents new methods to study nonlinear equation problems. Secondly, the work presented in this paper is the generalization and improvement of sum-type operator equation studied in [13], where $B$ is a null operator. Other particular cases of our research were 
investigated in [15], where $C$ is a null operator. Finally, we also discuss the solution of the nonlinear eigenvalue equation $A(x, x)+B(x, x)+C x=\lambda x$ and give dependency to the parameter. Hence, it is worthwhile to investigate the operator Eq. (1.1).

The content of this paper is organized as follows. In Sect. 2, we present some definitions, lemmas that will be used in the proofs of our theorems. In Sect. 3, we consider the unique existence of positive solutions for the operator Eq. (1.1) without assuming operators to be continuous and compact. In Sect. 4, we utilize the results obtained in Sect. 3 to study the existence and uniqueness of positive solutions for nonlinear fractional differential equation with multi-point fractional boundary conditions, and we also give a concrete example to illustrate our result.

\section{Preliminaries}

For convenience of the reader, we present some definitions, lemmas, and basic results that will be used in the proofs of our theorems. For more details, we refer the reader to [1-3, $24,25]$.

Definition 2.1 ([24]) Let $E$ be a real Banach space. A nonempty closed convex set $P \subset E$ is called a cone if it satisfies the following conditions:

(i) If $x \in P, \lambda \geq 0$, then $\lambda x \in P$;

(ii) If $x \in P$ and $-x \in P$, then $x=\theta$,

where $\theta$ denotes the zero element of $E$. The cone $P$ includes a partial order in $E$ given by

$$
x, y \in E, \quad x \leq y \quad \Leftrightarrow \quad y-x \in P,
$$

in which we denote $x<y$ or $y>x$ if $x \neq y$.

About the cone $P$, we also have the following definitions.

A cone $P$ is said to be solid if $\stackrel{\circ}{P}$ is nonempty, where $\stackrel{\circ}{P}=\{x \in P \mid x$ is an interior point of $P\}$.

A cone $P$ is called normal if there exists a constant $N>0$ such that, for all $x, y \in E$, $\theta \leq x \leq y$ implies $\|x\| \leq N\|y\|$, where $N$ is called the normality constant of $P$.

Given $h>\theta$ (i.e., $h \geq \theta$ and $h \neq \theta$ ), we denote by $P_{h}$ the set $P_{h}=\{x \in E \mid x \sim h\}$, in which $\sim$ is an equivalence relation, i.e., $x \sim y$ means that there exist $\lambda>0$ and $\mu>0$ such that $\lambda x \leq y \leq \mu x$ for all $x, y \in E$. It is easy to see that $P_{h} \subset P$ is convex and $\lambda P_{h}=P_{h}$ for all $\lambda>0$. If $\stackrel{\circ}{P} \neq \theta$ and $h \in \stackrel{P}{P}$, it is clear that $P_{h}=\stackrel{\circ}{P}$.

Definition 2.2 ([2]) An operator $A: P \times P \rightarrow P$ is said to be a mixed monotone operator if $A(x, y)$ is increasing in $x$ and decreasing in $y$, i.e., $\forall u_{i}, v_{i}(i=1,2) \in P, u_{1} \leq u_{2}, v_{1} \geq v_{2}$ imply $A\left(u_{1}, v_{1}\right) \leq A\left(u_{2}, v_{2}\right)$. An element $x \in P$ is called a fixed point of $A$ if $A(x, x)=x$.

Definition 2.3 ([25]) An operator $A: E \rightarrow E$ is said to be increasing if for any $x, y \in E$, $x \leq y$ implies $A x \leq A y$.

Definition 2.4 ([25]) An operator $A: P \rightarrow P$ is said to be sub-homogeneous if it satisfies

$$
A(t x) \geq t A x, \quad \forall t \in(0,1), x \in P .
$$


Definition 2.5 ([25]) Let $D=P$ or $D=\stackrel{\circ}{P}$ and $\alpha$ be a real number with $0 \leq \alpha<1$. An operator $A: D \rightarrow D$ is said to be $\alpha$-concave if it satisfies

$$
A(t x) \geq t^{\alpha} A x, \quad \forall t \in(0,1), x \in D .
$$

Definition 2.6 ([3]) Let $D$ be a convex subset in $E$. An operator $A: D \rightarrow E$ is called a convex operator (or concave operator) if

$$
A(t x+(1-t) y) \leq t A x+(1-t) A y \quad(\text { or } A(t x+(1-t) y) \geq t A x+(1-t) A y)
$$

for $x, y \in D$ with $y \leq x$ and every $t \in[0,1]$.

Lemma 2.1 ([9]) Let $P$ be a normal cone in E. Assume that $T: P \times P \rightarrow P$ is a mixed monotone operator and satisfies the following:

$\left(\mathrm{A}_{1}\right)$ There exists $h \in P$ with $h \neq \theta$ such that $T(h, h) \in P_{h}$;

$\left(\mathrm{A}_{2}\right)$ For any $u, v \in P$ and $t \in(0,1)$, there exists $\varphi(t) \in(t, 1]$ such that $T\left(t u, t^{-1} v\right) \geq$ $\varphi(t) T(u, v)$.

Then

(1) $T: P_{h} \times P_{h} \rightarrow P_{h}$;

(2) There exist $u_{0}, v_{0} \in P_{h}$ and $r \in(0,1)$ such that $r v_{0} \leq u_{0}<v_{0}$, $u_{0} \leq T\left(u_{0}, v_{0}\right) \leq T\left(v_{0}, u_{0}\right) \leq v_{0}$;

(3) $T$ has a unique fixed point $x^{*}$ in $P_{h}$;

(4) For any initial values $x_{0}, y_{0} \in P_{h}$, constructing successively the sequences

$$
x_{n}=T\left(x_{n-1}, y_{n-1}\right), \quad y_{n}=T\left(y_{n-1}, x_{n-1}\right), \quad n=1,2, \ldots,
$$

we have $x_{n} \rightarrow x^{*}$ and $y_{n} \rightarrow x^{*}$ as $n \rightarrow \infty$.

\section{Main results}

In this section, we study some fixed point theorems for a class of sum-type operators. This problem is equivalent to researching the existence and uniqueness of positive solutions for the operator equation $A(x, x)+B(x, x)+C x=x$, where $A, B$ are the mixed monotone operators with different properties, $C$ is an increasing operator. We assume that $E$ is a real Banach space with a partial order introduced by a normal cone $P$ of $E$. Take $h \in E, h>\theta$, $P_{h}$ is given as in the preliminaries.

Theorem 3.1 We will make the assumptions: $C: P \rightarrow P$ is an increasing sub-homogeneous operator, $A, B: P \times P \rightarrow P$ are two mixed monotone operators satisfying the following conditions:

$\left(\mathrm{L}_{1}\right)$ Let $\alpha \in(0,1)$ for any $t \in(0,1)$,

$$
A\left(t x, t^{-1} y\right) \geq t^{\alpha} A(x, y), \quad \forall x, y \in P
$$

$\left(\mathrm{L}_{2}\right)$ For any fixed $y \in P, B(\cdot, y): P \rightarrow P$ is concave, that is, for any $x_{1}, x_{2} \in P$ with $x_{2} \leq x_{1}$ and every $t \in(0,1)$, we have

$$
B\left(t x_{1}+(1-t) x_{2}, y\right) \geq t B\left(x_{1}, y\right)+(1-t) B\left(x_{2}, y\right)
$$


For any fixed $x \in P, B(x, \cdot): P \rightarrow P$ is convex, that is, for any $y_{1}, y_{2} \in P$ with $y_{2} \leq y_{1}$ and every $t \in(0,1)$, we have

$$
B\left(x, t y_{1}+(1-t) y_{2}\right) \leq t B\left(x, y_{1}\right)+(1-t) B\left(x, y_{2}\right)
$$

$\left(\mathrm{L}_{3}\right)$ There exists a constant $b \in\left[\frac{1}{2}, 1\right]$ such that

$$
B(\theta, l h) \geq b B(l h, \theta), \quad \forall l \geq 1
$$

$\left(\mathrm{L}_{4}\right)$ There is $h \in P, h>\theta$, such that $A(h, h) \in P_{h}, B(h, h) \in P_{h}$, and $C h \in P_{h}$.

$\left(\mathrm{L}_{5}\right)$ There exists a constant $\delta_{0}>0$ such that

$$
A(x, y) \geq \delta_{0}[B(x, y)+C x], \quad \forall x, y \in P .
$$

Then:

(1) $A: P_{h} \times P_{h} \rightarrow P_{h}, B: P_{h} \times P_{h} \rightarrow P_{h}, C: P_{h} \rightarrow P_{h}$;

(2) There exist $u_{0}, v_{0} \in P_{h}$ and $r \in(0,1)$ such that

$$
r v_{0} \leq u_{0}<v_{0}, \quad u_{0} \leq A\left(u_{0}, v_{0}\right)+B\left(u_{0}, v_{0}\right)+C u_{0} \leq A\left(v_{0}, u_{0}\right)+B\left(v_{0}, u_{0}\right)+C v_{0} \leq v_{0} ;
$$

(3) The operator equation $A(x, x)+B(x, x)+C x=x$ has a unique solution $x^{*}$ in $P_{h}$;

(4) For any initial values $x_{0}, y_{0} \in P_{h}$, constructing successively the sequences

$$
\begin{array}{ll}
x_{n}=A\left(x_{n-1}, y_{n-1}\right)+B\left(x_{n-1}, y_{n-1}\right)+C x_{n-1}, & n=1,2, \ldots, \\
y_{n}=A\left(y_{n-1}, x_{n-1}\right)+B\left(y_{n-1}, x_{n-1}\right)+C y_{n-1}, & n=1,2, \ldots,
\end{array}
$$

we have $x_{n} \rightarrow x^{*}$ and $y_{n} \rightarrow x^{*}$ as $n \rightarrow \infty$.

Proof The proof will be divided into two steps.

Step one: We demonstrate $A: P_{h} \times P_{h} \rightarrow P_{h}, B: P_{h} \times P_{h} \rightarrow P_{h}, C: P_{h} \rightarrow P_{h}$.

Firstly, for any $t \in(0,1), x, y \in P$, by (3.3) in $\left(\mathrm{L}_{2}\right)$, we have

$$
B(x, y)=B\left(x, t t^{-1} y+(1-t) \theta\right) \leq t B\left(x, t^{-1} y\right)+(1-t) B(x, \theta) .
$$

This clearly forces

$$
t B\left(x, t^{-1} y\right) \geq B(x, y)-(1-t) B(x, \theta) .
$$

By finding a sufficiently large constant $l \geq 1$ such that $x, y, t^{-1} y \leq l$. On account of $\left(\mathrm{L}_{3}\right)$, (3.2), (3.5), and the monotone property of operator $B$, we obtain

$$
\begin{aligned}
B\left(t x, t^{-1} y\right) & =B\left(t x+(1-t) \theta, t^{-1} y\right) \\
& \geq t B\left(x, t^{-1} y\right)+(1-t) B\left(\theta, t^{-1} y\right) \\
& \geq B(x, y)-(1-t) B(x, \theta)+(1-t) B\left(\theta, t^{-1} y\right) \\
& \geq B(x, y)+(1-t)[B(\theta, l h)-B(l h, \theta)]
\end{aligned}
$$




$$
\begin{aligned}
& \geq B(x, y)+(1-t)\left[B(\theta, l h)-\frac{1}{b} B(\theta, l h)\right] \\
& =B(x, y)+(1-t)\left(1-\frac{1}{b}\right) B(\theta, l h) \\
& \geq B(x, y)+(1-t)\left(1-\frac{1}{b}\right) B(x, y) \\
& =\left[\left(2-\frac{1}{b}\right)+\left(\frac{1}{b}-1\right) t\right] B(x, y) \geq t B(x, y), \quad \forall t \in(0,1), x, y \in P .
\end{aligned}
$$

That is,

$$
B\left(\frac{1}{t} x, t y\right) \leq \frac{1}{t} B(x, y), \quad \forall t \in(0,1), x, y \in P .
$$

Secondly, from (3.1) and (2.1), for any $t \in(0,1)$, we have

$$
\begin{aligned}
& A\left(\frac{1}{t} x, t y\right) \leq \frac{1}{t^{\alpha}} A(x, y), \quad \forall x, y \in P, \\
& C\left(\frac{1}{t} x\right) \leq \frac{1}{t} C x, \quad \forall x \in P .
\end{aligned}
$$

Since $A(h, h), B(h, h), C h \in P_{h}$, there exist positive constants $\mu_{i}, v_{i}(i=1,2,3)$ such that

$$
\mu_{1} h \leq A(h, h) \leq v_{1} h, \quad \mu_{2} h \leq B(h, h) \leq v_{2} h, \quad \mu_{3} h \leq C h \leq v_{3} h .
$$

For any $x, y \in P_{h}$, choose two sufficiently small numbers $c_{1}, c_{2} \in(0,1)$ such that

$$
c_{1} h \leq x \leq \frac{1}{c_{1}} h, \quad c_{2} h \leq y \leq \frac{1}{c_{2}} h .
$$

Set $c=\min \left\{c_{1}, c_{2}\right\}$, then $c \in(0,1)$, it follows from (3.1), (3.8), (3.10) that

$$
\begin{aligned}
& A(x, y) \leq A\left(\frac{1}{c_{1}} h, c_{2} h\right) \leq A\left(\frac{1}{c} h, c h\right) \leq \frac{1}{c^{\alpha}} A(h, h) \leq \frac{1}{c^{\alpha}} v_{1} h, \\
& A(x, y) \geq A\left(c_{1} h, \frac{1}{c_{2}} h\right) \geq A\left(c h, \frac{1}{c} h\right) \geq c^{\alpha} A(h, h) \geq c^{\alpha} \mu_{1} h .
\end{aligned}
$$

Noting that $\frac{1}{c^{\alpha}} \nu_{1}>0, c^{\alpha} \mu_{1}>0$, we can get $A(x, y) \in P_{h}$. That is, $A: P_{h} \times P_{h} \rightarrow P_{h}$.

Besides, by (3.6), (3.7), and (3.10), we deduce that

$$
\begin{aligned}
& B(x, y) \leq B\left(\frac{1}{c_{1}} h, c_{2} h\right) \leq B\left(\frac{1}{c} h, c h\right) \leq \frac{1}{c} B(h, h) \leq \frac{1}{c} v_{2} h, \\
& B(x, y) \geq B\left(c_{1} h, \frac{1}{c_{2}} h\right) \geq B\left(c h, \frac{1}{c} h\right) \geq c B(h, h) \geq c \mu_{2} h .
\end{aligned}
$$

Evidently, $\frac{1}{c} \nu_{2}>0, c \mu_{2}>0$. Thus $B(h, h) \in P_{h}$ implies $B: P_{h} \times P_{h} \rightarrow P_{h}$.

Moreover, according to (2.1), (3.9), and (3.10), we get

$$
C x \leq C\left(\frac{1}{c_{1}} h\right) \leq \frac{1}{c_{1}} C h \leq \frac{1}{c_{1}} v_{3} h,
$$




$$
C x \geq C\left(c_{1} h\right) \geq c_{1} C h \geq c_{1} \mu_{3} h .
$$

Clearly, $\frac{1}{c_{1}} v_{3}>0, c_{1} \mu_{3}>0$ show that $C x \in P_{h}$, which means $C: P_{h} \rightarrow P_{h}$. So conclusion (1) is founded.

Step two: We define an operator $T=A+B+C$ by

$$
T(x, y)=A(x, y)+B(x, y)+C x, \quad \forall x, y \in P .
$$

Then $T: P \times P \rightarrow P$ is a mixed monotone operator and $T(h, h) \in P_{h}$. Now we show that there exist $\varphi(t) \in(t, 1]$ such that

$$
T\left(t x, t^{-1} y\right) \geq \varphi(t) T(x, y), \quad \forall t \in(0,1), x, y \in P .
$$

Let $f$ be given by

$$
f(t)=\frac{t^{\beta}-t}{t^{\alpha}-t^{\beta}}, \quad \forall t \in(0,1), \beta \in(\alpha, 1) .
$$

Obviously, $f$ is increasing in $(0,1)$ and

$$
\lim _{t \rightarrow 0^{+}} f(t)=0, \quad \lim _{t \rightarrow 1^{-}} f(t)=\frac{1-\beta}{\beta-\alpha} .
$$

Also, fixing $t \in(0,1)$, we have

$$
\lim _{\beta \rightarrow 1^{-}} f(t)=\lim _{\beta \rightarrow 1^{-}} \frac{t^{\beta}-t}{t^{\alpha}-t^{\beta}}=0 .
$$

Hence there exists $\beta_{0}(t) \in(\alpha, 1)$ with respect to $t$ such that

$$
\frac{t^{\beta_{0}(t)}-t}{t^{\alpha}-t^{\beta_{0}(t)}} \leq \delta_{0}, \quad t \in(0,1)
$$

Accordingly, $\forall t \in(0,1), x, y \in P$, we have

$$
A(x, y) \geq \delta_{0}[B(x, y)+C x] \geq \frac{t^{\beta_{0}(t)}-t}{t^{\alpha}-t^{\beta_{0}(t)}}[B(x, y)+C x] .
$$

Then we get

$$
t^{\alpha} A(x, y)+t B(x, y)+t C x \geq t^{\beta_{0}(t)}[A(x, y)+B(x, y)+C x], \quad \forall t \in(0,1), x, y \in P .
$$

Consequently, by (3.12) we can obtain

$$
\begin{aligned}
T\left(t x, t^{-1} y\right) & =A\left(t x, t^{-1} y\right)+B\left(t x, t^{-1} y\right)+C(t x) \\
& \geq t^{\alpha} A(x, y)+t B(x, y)+t C x \\
& \geq t^{\beta_{0}(t)}[A(x, y)+B(x, y)+C x] \\
& =t^{\beta_{0}(t)} T(x, y), \quad \forall t \in(0,1), x, y \in P .
\end{aligned}
$$


Let $\varphi(t)=t^{\beta_{0}(t)}, t \in(0,1)$, then $\varphi(t) \in(t, 1]$, and

$$
T\left(t x, t^{-1} y\right) \geq \varphi(t) T(x, y), \quad \forall t \in(0,1), x, y \in P
$$

Combining step one and step two, we see at once that the operator $T$ satisfies all the conditions in Lemma 2.1. Having applied Lemma 2.1, we conclude that $\left(c_{1}\right)$ there exist $u_{0}, v_{0} \in P_{h}$ and $r \in(0,1)$ such that $r v_{0} \leq u_{0}<v_{0}, u_{0} \leq T\left(u_{0}, v_{0}\right) \leq T\left(v_{0}, u_{0}\right) \leq v_{0}$; $\left(c_{2}\right)$ the operator $T$ has a unique fixed point $x^{*}$ in $P_{h}$; $\left(c_{3}\right)$ for any initial values $x_{0}, y_{0} \in P_{h}$, constructing successively the sequences

$$
x_{n}=T\left(x_{n-1}, y_{n-1}\right), \quad y_{n}=T\left(y_{n-1}, x_{n-1}\right), \quad n=1,2, \ldots,
$$

we have $x_{n} \rightarrow x^{*}$ and $y_{n} \rightarrow x^{*}$ as $n \rightarrow \infty$. That is, conclusions (2)-(4) hold, which completes the proof.

By the proof of Theorem 3.1, we can obtain the following corollary.

Corollary 3.1 Suppose that $C: P \rightarrow P$ is an increasing sub-homogeneous operator, $A, B$ : $P \times P \rightarrow P$ are two mixed monotone operators and they satisfy conditions $\left(\mathrm{L}_{1}\right),\left(\mathrm{L}_{4}\right)$, and $\left(\mathrm{L}_{5}\right)$. Moreover, we assume that the operator B satisfies

$$
B\left(t x, t^{-1} y\right) \geq t B(x, y), \quad \forall t \in(0,1), x, y \in P .
$$

Then conclusions (1)-(4) in Theorem 3.1 also hold.

Corollary 3.2 Assume all the conditions about operators A, B, C in Theorem 3.1 or Corollary 3.1 hold. Then, for any given $\lambda>0$, the operator equation $A(x, x)+B(x, x)+C x=\lambda x$ has a unique positive solution $x_{\lambda}^{*} \in P_{h}$. Moreover, for any initial values $x_{0}, y_{0} \in P_{h}$, constructing successively the sequences

$$
\begin{aligned}
& x_{n}=\frac{1}{\lambda}\left[A\left(x_{n-1}, y_{n-1}\right)+B\left(x_{n-1}, y_{n-1}\right)+C x_{n-1}\right], \quad n=1,2, \ldots, \\
& y_{n}=\frac{1}{\lambda}\left[A\left(y_{n-1}, x_{n-1}\right)+B\left(y_{n-1}, x_{n-1}\right)+C y_{n-1}\right], \quad n=1,2, \ldots,
\end{aligned}
$$

we obtain $x_{n} \rightarrow x_{\lambda}^{*}$ and $y_{n} \rightarrow x_{\lambda}^{*}$ as $n \rightarrow \infty$.

Proof If we set operator $T=\lambda^{-1}(A+B+C)(\lambda>0)$, similar to the proof of Theorem 3.1, we can easily obtain that the operator $T$ satisfies all the conditions of Lemma 2.1 .

Corollary 3.3 Making the assumptions: $A, B: P \times P \rightarrow P$ are two mixed monotone operators and they satisfy $\left(\mathrm{L}_{1}\right)-\left(\mathrm{L}_{3}\right)$. Besides, we assume that:

$\left(\mathrm{L}_{4}^{\prime}\right)$ There is $h \in P, h>\theta$, such that $A(h, h) \in P_{h}$ and $B(h, h) \in P_{h}$.

$\left(\mathrm{L}_{5}^{\prime}\right)$ There exists a constant $\delta_{0}>0$ such that

$$
A(x, y) \geq \delta_{0} B(x, y), \quad \forall x, y \in P
$$

Then: 
(1) $A: P_{h} \times P_{h} \rightarrow P_{h}, B: P_{h} \times P_{h} \rightarrow P_{h}$;

(2) There exist $u_{0}, v_{0} \in P_{h}$ and $r \in(0,1)$ such that

$$
r v_{0} \leq u_{0}<v_{0}, \quad u_{0} \leq A\left(u_{0}, v_{0}\right)+B\left(u_{0}, v_{0}\right) \leq A\left(v_{0}, u_{0}\right)+B\left(v_{0}, u_{0}\right) \leq v_{0} ;
$$

(3) The operator equation $A(x, x)+B(x, x)=x$ has a unique solution $x^{*}$ in $P_{h}$;

(4) For any initial values $x_{0}, y_{0} \in P_{h}$, constructing successively the sequences

$$
\begin{array}{ll}
x_{n}=A\left(x_{n-1}, y_{n-1}\right)+B\left(x_{n-1}, y_{n-1}\right), & n=1,2, \ldots, \\
y_{n}=A\left(y_{n-1}, x_{n-1}\right)+B\left(y_{n-1}, x_{n-1}\right), & n=1,2, \ldots,
\end{array}
$$

we have $x_{n} \rightarrow x^{*}$ and $y_{n} \rightarrow x^{*}$ as $n \rightarrow \infty$.

Theorem 3.2 Assume $C: P \rightarrow P$ is an increasing $\alpha$-concave operator, $A, B: P \times P \rightarrow P$ are two mixed monotone operators. The operators satisfy conditions $\left(\mathrm{L}_{2}\right)-\left(\mathrm{L}_{4}\right)$ and the following hypotheses:

$\left(\mathrm{L}_{6}\right)$ For any $t \in(0,1)$,

$$
A\left(t x, t^{-1} y\right) \geq t A(x, y), \quad \forall x, y \in P .
$$

$\left(\mathrm{L}_{7}\right)$ There exists a constant $\delta_{0}>0$ such that

$$
A(x, y)+B(x, y) \leq \delta_{0} C x, \quad \forall x, y \in P
$$

Then conclusions (1)-(4) in Theorem 3.1 also hold true.

Proof In this section, the operator $B$ can be handled in much the same way as in Theorem 3.1, so it follows easily that $B: P_{h} \times P_{h} \rightarrow P_{h}$. Next, we only need to analyze the operators $A$ and $C$. By (3.13) and (2.2), it is obvious that

$$
\begin{aligned}
& A\left(\frac{1}{t} x, t y\right) \leq \frac{1}{t} A(x, y), \quad \forall t \in(0,1), x, y \in P . \\
& C\left(\frac{1}{t} x\right) \leq \frac{1}{t^{\alpha}} C x, \quad \forall x \in(0,1), x, y \in P .
\end{aligned}
$$

Similar to the analysis in the proof of Theorem 3.1, we deduce $A: P_{h} \times P_{h} \rightarrow P_{h}$ and $C$ : $P_{h} \rightarrow P_{h}$, which gives that conclusion (1) holds true.

Furthermore, we define the operator $T=A+B+C$ such that (3.11). Clearly, $T: P \times$ $P \rightarrow P$ is a mixed monotone operator and $T(h, h) \in P_{h}$. So condition $\left(A_{1}\right)$ in Lemma 2.1 is established. In the following, we only need to prove that there exist $\varphi(t) \in(t, 1]$ with respect to $t \in(0,1)$ such that

$$
T\left(t x, t^{-1} y\right) \geq \varphi(t) T(x, y), \quad \forall x, y \in P .
$$

In fact, we define an auxiliary function:

$$
f(t)=\frac{t^{\alpha}-t^{\beta}}{t^{\beta}-t}, \quad \forall t \in(0,1), \beta \in(\alpha, 1) .
$$


It is easily seen that $f$ is decreasing with respect to $t \in(0,1)$ and

$$
\lim _{t \rightarrow 0^{+}} f(t)=+\infty, \quad \lim _{t \rightarrow 1^{-}} f(t)=\frac{\beta-\alpha}{1-\beta} .
$$

Fix $t \in(0,1)$,

$$
\lim _{\beta \rightarrow 1^{-}} f(t)=\lim _{\beta \rightarrow 1^{-}} \frac{t^{\alpha}-t^{\beta}}{t^{\beta}-t}=+\infty
$$

We conclude from the above equations that there exists $\beta_{0}(t) \in(\alpha, 1)$ with respect to $t$ such that

$$
\frac{t^{\alpha}-t^{\beta_{0}(t)}}{t^{\beta_{0}(t)}-t} \geq \delta_{0}, \quad t \in(0,1)
$$

hence that

$$
A(x, y)+B(x, y) \leq \delta_{0} C x \leq \frac{t^{\alpha}-t^{\beta_{0}(t)}}{t^{\beta_{0}(t)}-t} C x, \quad \forall t \in(0,1), x, y \in P,
$$

and finally that

$$
t A(x, y)+t B(x, y)+t^{\alpha} C x \geq t^{\beta_{0}(t)}[A(x, y)+B(x, y)+C x], \quad \forall t \in(0,1), x, y \in P .
$$

In consequence, by (3.17), $\forall t \in(0,1), x, y \in P$, we have

$$
\begin{aligned}
T\left(t x, t^{-1} y\right) & =A\left(t x, t^{-1} y\right)+B\left(t x, t^{-1} y\right)+C(t x) \\
& \geq t A(x, y)+t B(x, y)+t^{\alpha} C x \\
& \geq t^{\beta_{0}(t)}[A(x, y)+B(x, y)+C x] \\
& =t^{\beta_{0}(t)} T(x, y) .
\end{aligned}
$$

Hence, condition $\left(A_{2}\right)$ in Lemma 2.1 holds true. An application of Lemma 2.1 implies that conclusions (2)-(4) hold true. The proof is completed.

Corollary 3.4 Let $\alpha \in(0,1) . C: P \rightarrow P$ is an increasing $\alpha$-concave operator, $B: P \times P \rightarrow P$ is a mixed monotone operator and satisfies conditions $\left(\mathrm{L}_{2}\right)-\left(\mathrm{L}_{3}\right)$. Furthermore, we assume that

$\left(\mathrm{L}_{6}^{\prime}\right)$ There is $h \in P, h>\theta$, such that $B(h, h) \in P_{h}$ and $C h \in P_{h}$.

$\left(\mathrm{L}_{7}^{\prime}\right)$ There exists a constant $\delta_{0}>0$ such that

$$
B(x, y) \leq \delta_{0} C x, \quad \forall x, y \in P
$$

Then:

(1) $B: P_{h} \times P_{h} \rightarrow P_{h}, C: P_{h} \rightarrow P_{h}$;

(2) There exist $u_{0}, v_{0} \in P_{h}$ and $r \in(0,1)$ such that

$$
r v_{0} \leq u_{0}<v_{0}, \quad u_{0} \leq B\left(u_{0}, v_{0}\right)+C u_{0} \leq B\left(v_{0}, u_{0}\right)+C v_{0} \leq v_{0}
$$


(3) The operator equation $B(x, x)+C x=x$ has a unique solution $x^{*}$ in $P_{h}$;

(4) For any initial values $x_{0}, y_{0} \in P_{h}$, constructing successively the sequences

$$
\begin{aligned}
& x_{n}=B\left(x_{n-1}, y_{n-1}\right)+C x_{n-1}, \quad n=1,2, \ldots, \\
& y_{n}=B\left(y_{n-1}, x_{n-1}\right)+C y_{n-1}, \quad n=1,2, \ldots,
\end{aligned}
$$

we have $x_{n} \rightarrow x^{*}$ and $y_{n} \rightarrow x^{*}$ as $n \rightarrow \infty$.

Corollary 3.5 Assume all the conditions about operators A, B, C in Theorem 3.2 hold. Then the operator equation $A(x, x)+B(x, x)+C x=\lambda x$ has a unique positive solution $x_{\lambda}^{*} \in P_{h}$ for any given $\lambda>0$. Besides, constructing successively the sequences

$$
\begin{array}{ll}
x_{n}=\frac{1}{\lambda}\left[A\left(x_{n-1}, y_{n-1}\right)+B\left(x_{n-1}, y_{n-1}\right)+C x_{n-1}\right], & n=1,2, \ldots, \\
y_{n}=\frac{1}{\lambda}\left[A\left(y_{n-1}, x_{n-1}\right)+B\left(y_{n-1}, x_{n-1}\right)+C y_{n-1}\right], & n=1,2, \ldots,
\end{array}
$$

for any initial values $x_{0}, y_{0} \in P_{h}$, we obtain $x_{n} \rightarrow x_{\lambda}^{*}$ and $y_{n} \rightarrow x_{\lambda}^{*}$ as $n \rightarrow \infty$.

In view of the fact that $P_{h}=\stackrel{\circ}{P}$ with $h \neq \theta$ and $h \in \stackrel{\circ}{P}$, we suppose that operators $A, B$ : $P_{h} \times P_{h} \rightarrow P_{h}, C: P_{h} \rightarrow P_{h}$ or $A, B: \stackrel{\circ}{P} \times \stackrel{\circ}{P} \rightarrow \stackrel{\circ}{P}, C: \stackrel{\circ}{P} \rightarrow \stackrel{\circ}{P}$ with $P$ is a solid cone, then $A(h, h) \in P_{h}, B(h, h) \in P_{h}$ and $C h \in P_{h}$ are automatically satisfied. So if we let $D=\stackrel{\circ}{P}$ or $P_{h}$, we have the following results.

Corollary 3.6 Let $C: D \rightarrow D$ be an increasing sub-homogeneous operator, $A, B: D \times D \rightarrow$ $D$ be two mixed monotone operators. Assume that

$\left(\mathrm{M}_{1}\right)$ Set $\alpha \in(0,1)$, for any $t \in(0,1)$,

$$
A\left(t x, t^{-1} y\right) \geq t^{\alpha} A(x, y), \quad \forall x, y \in D
$$

$\left(\mathrm{M}_{2}\right)$ For any fixed $y \in D, B(\cdot, y): D \rightarrow D$ is concave, for any fixed $x \in D, B(x, \cdot): D \rightarrow D$ is convex.

$\left(\mathrm{M}_{3}\right)$ There exists a constant $b \in\left[\frac{1}{2}, 1\right]$ such that

$$
B(\theta, l h) \geq b B(l h, \theta), \quad \forall l \geq 1 .
$$

$\left(\mathrm{M}_{4}\right)$ There exists a constant $\delta_{0}>0$ such that

$$
A(x, y) \geq \delta_{0}[B(x, y)+C x], \quad \forall x, y \in D
$$

Then conclusions (2)-(4) of Theorem 3.1 hold.

Corollary 3.7 Assume that $C: D \rightarrow D$ is an increasing $\alpha$-concave operator, $A, B: D \times D \rightarrow$ $D$ are two mixed monotone operators satisfying conditions $\left(\mathrm{M}_{2}\right)-\left(\mathrm{M}_{4}\right)$ and also meeting the following hypotheses:

$\left(\mathrm{M}_{5}\right)$ For any $t \in(0,1)$,

$$
A\left(t x, t^{-1} y\right) \geq t A(x, y), \quad \forall x, y \in D
$$


$\left(\mathrm{M}_{6}\right)$ There exists a constant $\delta_{0}>0$ such that

$$
A(x, y)+B(x, y) \leq \delta_{0} C x, \quad \forall x, y \in D
$$

Then conclusions (2)-(4) of Theorem 3.1 hold.

Remark 3.1 If we take the operator $B \equiv 0$ in Theorem 3.1, Theorem 3.2 and Corollaries 3.1-3.2, Corollaries 3.5-3.7, the corresponding results have been obtained by Zhai in [13]. In this case, our results generalize and improve some known results.

\section{Applications}

As the extensive development of the theory for fractional calculus itself, the fractional differential equations have been applied in many research fields, such as physics, chemistry, biology, control theory, economics, signal and image processing, etc. (see [26-29] and the references therein). Note that nonlinear operator theory has been proved to be a valuable tool in dealing with various differential and integral equations. Especially, fixed point method has been shown to be very useful in the study of the existence and uniqueness of solutions for differential equations. Such as in [11-18], the authors use the fixed point theorems of sum-type operators to study the fractional differential equations. In [30], Agarwal et al. apply Schauder's fixed point theorem, the upper and lower solution method, and topological degree theory to establish existence theory for unbounded solution. In [31], Becker et al. obtain positive solutions of an array of fractional differential equations by Schaefer's fixed point theorem. In [32], by means of fixed point methods combined with Karamata regular variation theory, Masmoudi et al. discuss existence, uniqueness, and boundary behavior of a positive solution to the nonlinear Sturm-Liouville problem. Motivated by the existing research, in this section we consider the following nonlinear fractional differential equation with the multi-point fractional boundary conditions:

$$
\left\{\begin{array}{l}
-D_{0^{+}}^{v} u(t)=f(t, u(t), u(t))+g(t, u(t), u(t))+h(t, u(t)), \quad 0<t<1 ; \\
u(0)=u^{\prime}(0)=\cdots u^{(n-2)}(0)=0 ; \\
\left.D_{0^{+}}^{p} u(t)\right|_{t=1}=\left.\sum_{i=1}^{m} a_{i} D_{0^{+}}^{q} u(t)\right|_{t=\xi_{i}},
\end{array}\right.
$$

where $D_{0^{+}}^{v}, D_{0^{+}}^{p}, D_{0^{+}}^{q}$ are the Riemann-Liouville fractional derivatives. The orders $v, p$, $q \in \mathbb{R}$, and they satisfy $v \in(n-1, n], p \in[1, n-2], q \in[0, p]$, and $n \in \mathbb{N}, n \geq 3$. $\xi_{i} \in \mathbb{R}$ for all $i=1,2, \ldots, m(m \in \mathbb{N}), 0<\xi_{1}<\cdots<\xi_{m}<1 . a_{i} \geq 0$ for all $i=1,2, \ldots, m(m \in \mathbb{N})$.

$$
\frac{1}{\Gamma(v-p)}-\frac{1}{\Gamma(v-q)} \sum_{i=1}^{m} a_{i} \xi_{i}^{v-q-1}>0 .
$$

By applying the fixed point theorems of sum-type operator obtained in Sect. 3, we focus on studying the existence and uniqueness of positive solution for problem (4.1), and also on constructing two sequences uniformly converging to the unique positive solution.

In the following, we will work in the Banach apace $E=C[0,1]=\{x:[0,1] \rightarrow \mathbb{R}$ is continuous $\}$ with the standard norm $\|x\|=\sup \{|x(t)|: t \in[0,1]\}$. Notice that this space can be equipped with a partial order given by

$$
x, y \in C[0,1], \quad x \leq y \quad \Leftrightarrow \quad x(t) \leq y(t) \text { for all } t \in[0,1] .
$$


Set $P=\{x \in C[0,1] \mid x(t) \geq 0, t \in[0,1]\}$, the standard cone. It is clear that $P$ is a normal cone in $E$ and the normality constant is 1 .

Definition 4.1 ([28]) Let $v>0$. Suppose that $u:(0,+\infty) \rightarrow \mathbb{R}$ is a continuous function. Then the Riemann-Liouville fractional derivative of order $v$ for the function $u$ is defined as

$$
D_{0^{+}}^{v} u(t)=\frac{1}{\Gamma(n-v)}\left(\frac{d}{d t}\right)^{n} \int_{0}^{t}(t-\tau)^{n-\nu-1} u(\tau) d \tau
$$

where $n=[v]+1$ with $[v]$ standing for the largest integer less than the number $v . \Gamma(v)$ is the Euler gamma function defined by

$$
\Gamma(v)=\int_{0}^{+\infty} t^{\nu-1} e^{-t} d t
$$

Lemma 4.1 (see Lemmas 1 and 2 in [33]) Assume that $\tilde{x} \in C[0,1] \cap L^{1}(0,1)$ is a continuous function. Then $u \in C[0,1]$ is the solution of the fractional boundary value problem:

$$
\begin{cases}-D_{0^{+}}^{v} u(t)=\widetilde{x}(t), & 0<t<1, n-1<v \leq n \\ u(0)=u^{\prime}(0)=\cdots u^{(n-2)}(0)=0, & n \in \mathbb{N}, n \geq 3 ; \\ \left.D_{0^{+}}^{p} u(t)\right|_{t=1}=\left.\sum_{i=1}^{m} a_{i} D_{0^{+}}^{q} u(t)\right|_{t=\xi_{i}}, & 1 \leq p \leq n-2,0 \leq q \leq p,\end{cases}
$$

where $a_{i} \geq 0, \xi_{i} \in \mathbb{R}$ for all $i=1,2, \ldots, m(m \in \mathbb{N})$ and $0<\xi_{1}<\cdots<\xi_{m}<1, \frac{1}{\Gamma(\nu-p)}-$ $\frac{1}{\Gamma(\nu-q)} \sum_{i=1}^{m} a_{i} \xi_{i}^{\nu-q-1}>0$, if and only if $u$ satisfies the integral equation

$$
u(t)=\int_{0}^{1} G(t, s) \widetilde{x}(s) d s, \quad t \in[0,1]
$$

where $G(t, s)$ is called the fractional Green function, which can be written as

$$
G(t, s)=g_{1}(t, s)+\frac{t^{\nu-1}}{\Delta} \sum_{i=1}^{m} a_{i} g_{2}\left(\xi_{i}, s\right), \quad \forall(t, s) \in[0,1] \times[0,1]
$$

with

$$
\begin{aligned}
& g_{1}(t, s)=\frac{1}{\Gamma(v)} \begin{cases}t^{\nu-1}(1-s)^{\nu-p-1}-(t-s)^{\nu-1}, & 0 \leq s \leq t \leq 1 ; \\
t^{\nu-1}(1-s)^{\nu-p-1}, & 0 \leq t \leq s \leq 1,\end{cases} \\
& g_{2}(t, s)=\frac{1}{\Gamma(v-q)} \begin{cases}t^{\nu-q-1}(1-s)^{\nu-p-1}-(t-s)^{\nu-q-1}, & 0 \leq s \leq t \leq 1 ; \\
t^{\nu-q-1}(1-s)^{\nu-p-1}, & 0 \leq t \leq s \leq 1,\end{cases}
\end{aligned}
$$

and

$$
\Delta=\frac{\Gamma(v)}{\Gamma(v-p)}-\frac{\Gamma(v)}{\Gamma(v-q)} \sum_{i=1}^{m} a_{i} \xi_{i}^{\nu-q-1}>0
$$


By Lemmas 3 and 4 in [33], we can easily obtain that the Green function $G(t, s)$ defined by (4.2) has the following properties.

Lemma 4.2 The function $G(t, s)$ satisfies the following conditions:

(1) $G(t, s)$ is continuous on the unit square $[0,1] \times[0,1]$;

(2) $G(t, s) \geq 0$ for each $(t, s) \in[0,1] \times[0,1]$;

(3) $t^{\nu-1} J(s) \leq G(t, s) \leq \sigma t^{\nu-1}, \quad \forall t, s \in[0,1]$,

where

$$
J(s)=(1-s)^{\nu-p-1} \frac{1-(1-s)^{p}}{\Gamma(v)}+\frac{1}{\Delta} \sum_{i=1}^{m} a_{i} g_{2}\left(\xi_{i}, s\right), \quad s \in[0,1],
$$

with $\Delta$ defined in (4.3).

$$
\sigma=\frac{1}{\Gamma(v)}+\frac{1}{\Delta \Gamma(v-q)} \sum_{i=1}^{m} a_{i} \xi_{i}^{v-q-1}
$$

Theorem 4.1 Assume the following conditions:

$\left(H_{1}\right) f, g:[0,1] \times[0,+\infty) \times[0,+\infty) \rightarrow[0,+\infty)$ and $h:[0,1] \times[0,+\infty) \rightarrow[0,+\infty)$ are continuous with $f(t, 0,1) \not \equiv 0, g(t, 0,1) \not \equiv 0$, and $h(t, 0) \not \equiv 0$;

$\left(H_{2}\right) f(t, u, v), g(t, u, v)$ are increasing in $u \in[0,+\infty)$ for fixed $t \in[0,1]$ and $v \in[0,+\infty)$, decreasing in $v \in[0,+\infty)$ for fixed $t \in[0,1]$ and $u \in[0,+\infty) ; g(t, u)$ is increasing in $u \in[0,+\infty)$ for fixed $t \in[0,1]$;

$\left(H_{3}\right)$ For any $\lambda \in(0,1)$, there exists a constant $\alpha \in(0,1)$ such that $\forall t \in[0,1], u, v \in$ $[0,+\infty), f\left(t, \lambda u, \lambda^{-1} v\right) \geq \lambda^{\alpha} f(t, u, v) ;$ for fixed $t \in[0,1], v \in[0,+\infty), g(t, \cdot, v)$ is concave, for fixed $t \in[0,1], u \in[0,+\infty), g(t, u, \cdot)$ is convex; and for all $\lambda \in(0,1)$, $\forall t \in[0,1], u \in[0,+\infty), h(t, \lambda u) \geq \lambda h(t, u) ;$

$\left(H_{4}\right)$ There exists $\frac{1}{2} \leq b \leq 1$ such that $g(t, 0, \operatorname{lh}(t)) \geq b g(t, \operatorname{lh}(t), 0), \forall l \geq 1$;

$\left(H_{5}\right)$ There exists a constant $\delta_{0}>0$ such that $f(t, u, v) \geq \delta_{0}(g(t, u, v)+h(t, u)), t \in[0,1]$, $u, v \in[0,+\infty)$.

Then:

(1) There exist $u_{0}, v_{0} \in P_{h}$ and $r \in(0,1)$ such that $r v_{0} \leq u_{0}<v_{0}$ and

$$
\begin{cases}u_{0}(t) \leq \int_{0}^{1} G(t, s)\left[f\left(s, u_{0}(s), v_{0}(s)\right)+g\left(s, u_{0}(s), v_{0}(s)\right)+h\left(s, u_{0}(s)\right)\right] d s, & t \in[0,1], \\ v_{0}(t) \geq \int_{0}^{1} G(t, s)\left[f\left(s, v_{0}(s), u_{0}(s)\right)+g\left(s, v_{0}(s), u_{0}(s)\right)+h\left(s, v_{0}(s)\right)\right] d s, & t \in[0,1]\end{cases}
$$

where $h(t)=t^{\alpha-1}, t \in[0,1]$;

(2) Problem (4.1) has a unique positive solution $u^{*}$ in $P_{h}$;

(3) For any $x_{0}, y_{0} \in P_{h}$, constructing successively the sequences

$$
\begin{cases}x_{n+1}(t)=\int_{0}^{1} G(t, s)\left[f\left(s, x_{n}(s), y_{n}(s)\right)+g\left(s, x_{n}(s), y_{n}(s)\right)+h\left(s, x_{n}(s)\right)\right] d s, & n=0,1,2, \ldots \\ y_{n+1}(t)=\int_{0}^{1} G(t, s)\left[f\left(s, y_{n}(s), x_{n}(s)\right)+g\left(s, y_{n}(s), x_{n}(s)\right)+h\left(s, y_{n}(s)\right)\right] d s, & n=0,1,2, \ldots\end{cases}
$$

we have both $x_{n}(t)$ and $y_{n}(t)$ converge uniformly to $u^{*}(t)$ for all $t \in[0,1]$. 
Proof To begin with, we define three operators $A: P \times P \rightarrow E ; B: P \times P \rightarrow E ; C: P \rightarrow E$ by

$$
\begin{aligned}
& A(u, v)(t)=\int_{0}^{1} G(t, s) f(s, u(s), v(s)) d s, \quad B(u, v)(t)=\int_{0}^{1} G(t, s) g(s, u(s), v(s)) d s, \\
& C u(t)=\int_{0}^{1} G(t, s) h(s, u(s)) d s .
\end{aligned}
$$

Set $\widetilde{x}(t)=f(t, u(t), u(t))+g(t, u(t), u(t))+h(t, u(t))$ in Lemma 4.1, it is easy to prove that $u$ is the solution of problem (4.1) if and only if it is a fixed point of the operator equation $u=A(u, u)+B(u, u)+C u$. In the sequel, we check that $A, B, C$ satisfy all the assumptions of Theorem 3.1.

Firstly, it follows from $\left(H_{1}\right)$ and the fact $G(t, s) \geq 0, \forall t, s \in[0,1]$ that $A, B: P \times P \rightarrow P$ and $C: P \rightarrow P$. Further, by $\left(H_{2}\right)$, we can easily obtain $A, B$ are two monotone operators, and $C$ is an increasing operator.

Secondly, we show that $A, B$ satisfy conditions (3.1)-(3.3) and $C$ is a sub-homogeneous operator. In fact, for any $\lambda \in(0,1)$ and $u, v \in P$, by $\left(H_{3}\right)$ we have

$$
\begin{aligned}
A\left(\lambda u, \lambda^{-1} v\right)(t) & =\int_{0}^{1} G(t, s) f\left(s, \lambda u(s), \lambda^{-1} v(s)\right) d s \\
& \geq \lambda^{\alpha} \int_{0}^{1} G(t, s) f(s, u(s), v(s)) d s=\lambda^{\alpha} A(u, v)(t),
\end{aligned}
$$

that is $A\left(\lambda u, \lambda^{-1} v\right) \geq \lambda^{\alpha} A(u, v)$ for $\lambda \in(0,1), u, v \in P$, which satisfies (3.1).

Also, for fixed $t \in(0,1), v \in P$, for any $a \in(0,1), u_{1}, u_{2} \in P$,

$$
\begin{aligned}
B & \left(a u_{1}+(1-a) u_{2}, v\right)(t) \\
\quad & =\int_{0}^{1} G(t, s) g\left(s, a u_{1}(s)+(1-a) u_{2}(s), v(s)\right) d s \\
\quad & \int_{0}^{1} G(t, s)\left[a g\left(s, u_{1}(s), v(s)\right)+(1-a) g\left(s, u_{2}(s), v(s)\right)\right] d s \\
\quad & a \int_{0}^{1} G(t, s) g\left(s, u_{1}(s), v(s)\right) d s+(1-a) \int_{0}^{1} G(t, s) g\left(s, u_{2}(s), v(s)\right) d s \\
& =a B\left(u_{1}, v\right)(t)+(1-a) B\left(u_{2}, v\right)(t),
\end{aligned}
$$

which means $B\left(a u_{1}+(1-a) u_{2}, v\right) \geq a B\left(u_{1}, v\right)+(1-a) B\left(u_{2}, v\right)$. So, for fixed $v \in P, B(t, \cdot, v)$ : $P \rightarrow P$ is concave. Besides, for any $a \in(0,1), v_{1}, v_{2} \in P$,

$$
\begin{aligned}
B\left(u, a v_{1}+(1-a) v_{2}\right)(t) \\
\quad=\int_{0}^{1} G(t, s) g\left(s, u(s), a v_{1}(s)+(1-a) v_{2}(s)\right) d s \\
\quad \leq \int_{0}^{1} G(t, s)\left[a g\left(s, u(s), v_{1}(s)\right)+(1-a) g\left(s, u(s), v_{2}(s)\right)\right] d s \\
\quad=a \int_{0}^{1} G(t, s) g\left(s, u(s), v_{1}(s)\right) d s+(1-a) \int_{0}^{1} G(t, s) g\left(s, u(s), v_{2}(s)\right) d s \\
\quad=a B\left(u, v_{1}\right)(t)+(1-a) B\left(u, v_{2}\right)(t),
\end{aligned}
$$


which implies $B\left(u, a v_{1}+(1-a) v_{2}\right) \leq a B\left(u, v_{1}\right)+(1-a) B\left(u, v_{2}\right)$, hence for fixed $u \in P$, $C(t, u, \cdot): P \rightarrow P$ is convex and satisfies (3.3). Furthermore, for any $\lambda \in(0,1), u \in P$, from $\left(H_{3}\right)$ we know that

$$
C(\lambda u)(t)=\int_{0}^{1} G(t, s) h(s, \lambda u(s)) d s \geq \lambda \int_{0}^{1} G(t, s) h(s, u(s)) d s=\lambda C u(t),
$$

that is, $C(\lambda u) \geq \lambda C u$ for $\lambda \in(0,1), u \in P$ and the operator $C$ is sub-homogeneous.

Thirdly, we prove that condition $\left(L_{3}\right)$ of Theorem 3.1 is satisfied. From $\left(H_{4}\right)$, there exists $b \in\left[\frac{1}{2}, 1\right]$ such that

$$
\begin{aligned}
B(\theta, \operatorname{lh})(t) & =\int_{0}^{1} G(t, s) g(s, 0, \operatorname{lh}(s)) d s \\
& \geq b \int_{0}^{1} G(t, s) g(s, \operatorname{lh}(s), 0) d s=b B(\operatorname{lh}, \theta)(t), \quad \forall l \geq 1 .
\end{aligned}
$$

Fourthly, we show that $A(h, h) \in P_{h}, B(h, h) \in P_{h}$ and $C h \in P_{h}$. Let $h \in P_{h}$ be defined by $h(t)=t^{\nu-1}, t \in[0,1]$. We can obtain that $h(t) \in[0,1]$. From $\left(H_{2}\right)$ and Lemma 4.2, we deduce

$$
\begin{aligned}
& A(h, h)(t)=\int_{0}^{1} G(t, s) f\left(s, s^{\nu-1}, s^{\nu-1}\right) d s \geq h(t) \int_{0}^{1} J(s) f(s, 0,1) d s \\
& A(h, h)(t)=\int_{0}^{1} G(t, s) f\left(s, s^{\nu-1}, s^{\nu-1}\right) d s \leq \sigma h(t) \int_{0}^{1} f(s, 1,0) d s
\end{aligned}
$$

where $J(s)$ and $\sigma$ are defined by (4.4), (4.5). Denote

$$
c_{1}=\int_{0}^{1} J(s) f(s, 0,1) d s, \quad c_{2}=\sigma \int_{0}^{1} f(s, 1,0) d s .
$$

Then we have $c_{1} h \leq A(h, h) \leq c_{2} h$. It follows from $\left(H_{2}\right),\left(H_{1}\right)$ that

$$
f(s, 1,0) \geq f(s, 0,1) \geq 0, \quad \forall s \in[0,1] .
$$

Since $f(t, 0,1) \not \equiv 0$, we get

$$
\int_{0}^{1} f(s, 1,0) d s \geq \int_{0}^{1} f(s, 0,1) d s>0 .
$$

Hence, we have $c_{1}>0, c_{2}>0$, which means $A(h, h) \in P_{h}$.

Similarly,

$$
\begin{aligned}
& h(t) \int_{0}^{1} J(s) g(s, 0,1) d s \leq B(h, h) \leq \sigma h(t) \int_{0}^{1} g(s, 1,0) d s, \\
& h(t) \int_{0}^{1} J(s) h(s, 0) d s \leq C h \leq \sigma h(t) \int_{0}^{1} h(s, 1) d s .
\end{aligned}
$$

According to the fact that $g(t, 0,1) \not \equiv, h(t, 0) \not \equiv 0$, we easily prove $B(h, h) \in P_{h}, C h \in P_{h}$. As a result, condition $\left(L_{4}\right)$ of Theorem 3.1 is satisfied. 
Lastly, we show that condition $\left(L_{5}\right)$ of Theorem 3.1 holds true for $u, v \in P$ and any $t \in$ $[0,1]$. From $\left(H_{5}\right)$, we derive that

$$
\begin{aligned}
A(u, v)(t) & =\int_{0}^{1} G(t, s) f(s, u(s), v(s)) d s \geq \delta_{0} \int_{0}^{1} G(t, s)(g(s, u(s), v(s))+h(s, u(s))) d s \\
& =\delta_{0}\left[\int_{0}^{1} G(t, s) g(s, u(s), v(s)) d s+\int_{0}^{1} G(t, s) h(s, u(s)) d s\right] \\
& =\delta_{0}[B(u, v)(t)+(C u)(t)],
\end{aligned}
$$

then we get $A(u, v) \geq \delta_{0}(B(u, v)+C u)$ for $u, v \in P$. So all the conditions of Theorem 3.1 are satisfied. An application of Theorem 3.1 implies that problem (4.1) has a unique positive solution $u^{*}$ in $P_{h}$ and also conclusions (1) and (3) in Theorem 4.1 hold true.

By using Theorem 3.2, we can easily prove the following result.

Theorem 4.2 Assume that $\left(H_{1}\right),\left(H_{2}\right)$, and $\left(H_{4}\right)$ hold, and the following conditions are also satisfied.

$\left(H_{6}\right) f\left(t, \lambda u, \lambda^{-1} v\right) \geq \lambda f(t, u, v), \forall t \in[0,1], \lambda \in(0,1), u, v \in[0,+\infty)$; for fixed $t \in[0,1]$, $v \in[0,+\infty), g(t, \cdot, v)$ is concave, for fixed $t \in[0,1], u \in[0,+\infty), g(t, u, \cdot)$ is convex; and there exists a constant $\alpha \in(0,1)$ such that $h(t, \lambda u) \geq \lambda^{\alpha} h(t, u)$ for $\lambda \in(0,1), t \in[0,1], u \in[0,+\infty)$;

$\left(H_{7}\right)$ There exists a constant $\delta_{0}>0$ such that $f(t, u, v)+g(t, u, v) \leq \delta_{0} h(t, u), \forall t \in[0,1]$, $u, v \in[0,+\infty)$.

Then, conclusions (1)-(3) of Theorem 4.1 still hold.

In what follows, we give a concrete example to illustrate our main result.

Example 4.1 Consider the following problem:

$$
\left\{\begin{array}{l}
-D_{0^{+}}^{\frac{17}{4}} u(t)=(u(t)+1)^{\frac{1}{2}}+(u(t)+1)^{-\frac{1}{2}}+\frac{1}{3} e^{-u(t)}-\frac{1}{3} e^{-u(t)}+\frac{u(t)}{1+u(t)} s(t)-2 t^{2}+6, \\
0<t<1 ; \\
u(0)=u^{\prime}(0)=u^{\prime \prime}(0)=u^{\prime \prime \prime}(0)=0 \\
\left.D_{0^{+}}^{\frac{5}{2}} u(t)\right|_{t=1}=\left.D_{0^{+}}^{\frac{9}{4}} u(t)\right|_{t=\frac{1}{5}}+\left.\frac{1}{2} D_{0^{+}}^{\frac{9}{4}} u(t)\right|_{t=\frac{2}{5}}+\left.\frac{1}{3} D_{0^{+}}^{\frac{9}{4}} u(t)\right|_{t=\frac{3}{5}}+\left.\frac{1}{4} D_{0^{+}}^{\frac{9}{4}} u(t)\right|_{t=\frac{4}{5}},
\end{array}\right.
$$

where

$$
\begin{aligned}
& n=5, \quad v=\frac{17}{4}, \quad p=\frac{5}{2}, \quad q=\frac{9}{4}, \quad m=4, \quad \xi_{1}=\frac{1}{5}, \quad \xi_{2}=\frac{2}{5}, \\
& \xi_{3}=\frac{3}{5}, \quad \xi_{4}=\frac{4}{5}, \quad a_{1}=1, \quad a_{2}=\frac{1}{2}, \quad a_{3}=\frac{1}{3}, \quad a_{4}=\frac{1}{4}, \\
& \frac{1}{\Gamma(v-p)}-\frac{1}{\Gamma(v-q)} \sum_{i=1}^{m} a_{i} \xi_{i}^{v-q-1}=\frac{1}{\Gamma\left(\frac{7}{4}\right)}-\frac{1}{\Gamma(2)} \cdot \frac{4}{5}=0.288065>0,
\end{aligned}
$$

and $s:[0,1] \rightarrow[0,+\infty)$ is continuous with $s \neq 0$. Here we set $s_{\max }=\max \{s(t): t \in[0,1]\}>0$.

This example can be written in the form of (4.1) with the functions $f, g, h$ defined by

$$
f(t, x, y)=(x+1)^{\frac{1}{2}}+(y+1)^{-\frac{1}{2}}+3-t^{2}
$$




$$
g(t, x, y)=\frac{1}{3} e^{-y}-\frac{1}{3} e^{-x}+2-t^{2}, \quad h(t, x)=\frac{x}{1+x} s(t)+1
$$

Next, we show that all the conditions of Theorem 4.1 are satisfied.

(1) $f, g:[0,1] \times[0,+\infty) \times[0,+\infty) \rightarrow[0,+\infty)$ and $h:[0,1] \times[0,+\infty) \rightarrow[0,+\infty)$ are continuous with $f(t, 0,1)=4+2^{-\frac{1}{2}}-t^{2} \not \equiv 0, g(t, 0,1)=\frac{5}{3}+\frac{1}{3 e}-t^{2} \not \equiv 0, h(t, 0)=1 \neq 0$.

(2) Obviously, $f(t, x, y), g(t, x, y)$ are increasing in $x \in[0,+\infty)$ for fixed $t \in[0,1]$ and $y \in[0,+\infty)$, decreasing in $y \in[0,+\infty)$ for fixed $t \in[0,1]$ and $x \in[0,+\infty)$, and $h(t, x)$ is increasing in $x \in[0,+\infty)$ for fixed $t \in[0,1]$.

(3) For $\lambda \in(0,1), t \in[0,1], x, y \in[0,+\infty)$, taking $\alpha=\frac{1}{2}$, we have

$$
\begin{aligned}
f\left(t, \lambda x, \lambda^{-1} y\right) & =(\lambda x+1)^{\frac{1}{2}}+\left(\lambda^{-1} y+1\right)^{-\frac{1}{2}}+3-t^{2} \\
& \geq \lambda^{\frac{1}{2}}\left((x+1)^{\frac{1}{2}}+(y+1)^{-\frac{1}{2}}+3-t^{2}\right)=\lambda^{\alpha} f(t, x, y) .
\end{aligned}
$$

For all $\lambda \in(0,1), t \in[0,1]$, and $x \in[0,+\infty)$, we deduce

$$
h(t, \lambda x)=\frac{\lambda x}{1+\lambda x} s(t)+1 \geq \frac{\lambda x}{1+x} s(t)+\lambda=\lambda h(t, x) .
$$

Besides, we can easily obtain

$$
g_{x x}^{\prime \prime}(t, x, y)=-\frac{1}{3} e^{-x}<0, \quad g_{y y}^{\prime \prime}(t, x, y)=\frac{1}{3} e^{-y}>0 .
$$

So, for fixed $t \in[0,1], y \in[0,+\infty), g(t, \cdot y)$ is concave; for fixed $t \in(0,1), x \in[0,+\infty)$, $g(t, x, \cdot)$ is convex.

(4) For all $s \in[0,1], y \in[0,+\infty)$, taking $b=\frac{1}{2}$, we derive

$$
g(s, 0, y)=\left(1-s^{2}\right)+\left(\frac{2}{3}+\frac{1}{3} e^{-y}\right) \geq \frac{1}{2}\left[\left(1-s^{2}\right)+\left(\frac{4}{3}-\frac{1}{3} e^{-y}\right)\right]=g(s, y, 0) .
$$

(5) If we take $\delta_{0}=\min \left\{\frac{2}{3\left(s_{\max }+1\right)}, 1\right\}$, then we obtain

$$
\begin{aligned}
f(t, x, y) & =(x+1)^{\frac{1}{2}}+(y+1)^{-\frac{1}{2}}+3-t^{2} \\
& \geq(x+1)^{\frac{1}{2}}+(y+1)^{-\frac{1}{2}}+\frac{2}{3}+\frac{1}{3} e^{-y}-\frac{1}{3} e^{-x}+2-t^{2} \\
& \geq \frac{2}{3\left(s_{\max }+1\right)} \cdot\left(s_{\max }+1\right)+\frac{1}{3} e^{-y}-\frac{1}{3} e^{-x}+2-t^{2} \\
& \geq \frac{2}{3\left(s_{\max }+1\right)} \cdot\left(\frac{x}{1+x} s(t)+1\right)+\frac{1}{3} e^{-y}-\frac{1}{3} e^{-x}+2-t^{2} \\
& \geq \delta_{0}\left(\frac{x}{1+x} s(t)+1+\frac{1}{3} e^{-y}-\frac{1}{3} e^{-x}+2-t^{2}\right) \\
& =\delta_{0}(g(t, x, y)+h(t, x)) \quad \forall t \in[0,1], x, y \in[0,+\infty) .
\end{aligned}
$$

Hence all the conditions of Theorem 4.1 are satisfied. An application of Theorem 4.1 implies that problem (4.6) has a unique positive solution in $P_{h}$, where $h(t)=t^{\nu-1}=t^{\frac{13}{4}}$, $t \in[0,1]$. 


\title{
5 Conclusions
}

In this paper, we investigate a class of nonlinear sum-type operators without considering the existence of upper-lower solutions or compactness or continuity. The sufficient conditions have been established for such sum-type operators to have a unique positive fixed point in $P_{h}$, and two iterative sequences are also constructed to converge to the fixed point. Further, we apply the obtained results to prove the existence and uniqueness of positive solutions for a nonlinear fractional differential equation with multi-point fractional boundary conditions. The main contribution is that we provide a new method to deal with the unique positive solution of the nonlinear differential equations. Our study enriches the fixed point theorems of nonlinear sum-type operators.

\author{
Acknowledgements \\ We are thankful to the editor and the anonymous reviewers for many valuable suggestions to improve this paper. \\ Funding \\ This project is supported by the Key Research and Development Plan of Shanxi Province (No. 201703D221031-1) and \\ Innovation Project of Shanxi Postgraduate Education (No. 2017BY039).
}

Availability of data and materials

Data sharing not applicable to this article as no datasets were generated or analysed during the current study.

Competing interests

The authors declare that they have no competing interests.

\section{Authors' contributions}

HW participated in the design of the study and drafted the manuscript. LZ and XW carried out the theoretical studies and helped to draft the manuscript. All authors read and approved the final manuscript.

\section{Author details}

${ }^{1}$ College of Mathematics, Taiyuan University of Technology, Taiyuan, P.R. China. ${ }^{2}$ Department of Scientific Computing, Florida State University, Tallahassee, USA.

\section{Publisher's Note}

Springer Nature remains neutral with regard to jurisdictional claims in published maps and institutional affiliations.

Received: 2 May 2018 Accepted: 5 September 2018 Published online: 18 September 2018

\section{References}

1. Zhao, Z: Existence and uniqueness of fixed points for some mixed monotone operators. Nonlinear Anal. 73, $1481-1490(2010)$

2. Guo, D., Lakshmikantham, V.: Coupled fixed points of nonlinear operators with applications. Nonlinear Anal. 11, 623-632 (1987)

3. Amann, H.: On the number of solutions of nonlinear equations in ordered Banach spaces. J. Funct. Anal. 11, 346-384 (1972)

4. Li, K., Liang, J., Xiao, T.: A fixed point theorem for convex and decreasing operators. Nonlinear Anal. 63, 209-216 (2005)

5. Zhao, Z., Du, X.: Fixed points of generalized e-concave (generalized e-convex) operators and their applications. J. Math. Anal. Appl. 334, 1426-1438 (2007)

6. Zhai, C., Yang, C., Guo, C.: Positive solutions of operator equations on ordered Banach spaces and appliccations. Comput. Math. Appl. 56, 3150-3156 (2008)

7. Zhai, C., Cao, X.: Fixed point theorems for $\tau$ - $\varphi$-concave operators and applications. Comput. Math. Appl. 59, 532-538 (2010)

8. Zhao, Z: Fixed point of $\tau$ - $\varphi$-convex operators and applications. Appl. Math. Lett. 23, 561-566 (2010)

9. Zhai, C., Zhang, L.: New fixed point theorems for mixed monotone operators and local existence-uniqueness of positive solutions for nonlinear boundary value problems. J. Math. Anal. Appl. 382, 594-614 (2011)

10. Zhai, C., Wang, L.: $\varphi$-(h,e)-concave operators and applications. J. Math. Anal. Appl. 454, 571-584 (2017)

11. Sang, Y.: Existence and uniqueness of fixed points for mixed monotone operator with perturbations. Electron. J. Differ. Equ. 2013, 233 (2013)

12. Amor, S.H.: Positive solutions for some boundary value problems via a new fixed point theorem. Positivity 19 , 587-602 (2015)

13. Zhai, C., Hao, M.: Fixed point theorems for mixed monotone operator with perturbation and application to fractional differential equation boundary value problems. Nonlinear Anal. 75, 2542-2551 (2012)

14. Zhai, C., Anderson, D.R.: A sum operator equation and applications to nonlinear elastic beam equations and Lane-Emden-Fowler equations. J. Math. Anal. Appl. 375, 388-400 (2011)

15. Zhang, X., Liu, L., Wu, Y.: Fixed point theorems for the sum of three classes of mixed monotone operators and applications. Fixed Point Theory Appl. 2016, 49 (2016) 
16. Liu, L., Zhang, X., Jiang, J., Wu, Y.: The unique solution of a class of sum mixed monotone operator equations and its application to fractional boundary value problems. J. Nonlinear Sci. Appl. 9, 2943-2958 (2016)

17. Wang, H., Zhang, L.: The solution for a class of sum operator equation and its application to fractional differential equation boundary value problems. Bound. Value Probl. 2015, 203 (2015)

18. Yang, C., Zhai, C., Hao, M.: Uniqueness of positive solutions for several classes of sum operator equations and applications. J. Inequal. Appl. 2014, 58 (2014)

19. Azman, I., Jelia, M., López, B., Sadarangani, K., Samet, B.: Positive solutions for a class of fractional boundary value problems with fractional boundary conditions. J. Nonlinear Sci. Appl. 11, 237-251 (2018)

20. Cabrera, I., López, B., Sadarangani, K.: Existence of positive solutions for the nonlinear elastic beam equation via a mixed monotone operator. J. Comput. Appl. Math. 327, 306-313 (2018)

21. Feng, H., Zhai, C.: Existence and uniqueness of positive solutions for a class of fractional differential equation with integral boundary conditions. Nonlinear Anal., Model. Control 22, 160-172 (2017)

22. Liu, L., Li, H., Liu, C., Wu, Y.: Existence and uniqueness of positive solutions for singular fractional differential systems with coupled integral boundary conditions. J. Nonlinear Sci. Appl. 10, 243-262 (2017)

23. Min, D., Liu, L., Wu, Y.: Uniqueness of positive solutions for the singular fractional differential equations involving integral boundary value conditions. Bound. Value Probl. 2018, 23 (2018)

24. Deimling, K.: Nonlinear Functional Analysis. Springer, Berlin (1985)

25. Guo, D., Lakshmikantham, V:: Nonlinear Problems in Abstract Cones. Academic Press, New York (1988)

26. Losa, G., Merlini, D., Nonnenmacher, T., Weibel, E.: Fractals in Biology and Medicine. Birkhäuser, Basel (1998)

27. Hilfer, R.: Applications of Fractional Calculus in Physics. World Scientific, Singapore (2000)

28. Lakshmikantham, V., Vatsala, A.: Basic theory of fractional differential equations. Nonlinear Anal. 69, 2677-2682 (2008)

29. Kilbas, A., Srivastava, H., Trujillo, J.: Theory and Applications of Fractional Differential Equations. Elsevier, Amsterdam (2006)

30. Agarwal, R.P., Cetin, E.: Unbounded solutions of third order three-point boundary value problems on a half-line. Adv. Nonlinear Anal. 5, 105-119 (2016)

31. Becker, L.C., Burton, T.A., Purnaras, I.K.: Integral and fractional equations, positive solutions, and Schaefer's fixed point theorem. Opusc. Math. 36, 431-458 (2016)

32. Masmoudi, S., Zermani, S.: Existence and boundary behavior of positive solutions for a Sturm-Liouville problem. Opusc. Math. 36, 613-629 (2016)

33. Henderson, J., Luca, R.: Existence of positive solutions for a singular fractional boundary value problem. Nonlinear Anal., Model. Control 22, 99-114 (2017)

\section{Submit your manuscript to a SpringerOpen ${ }^{\circ}$ journal and benefit from:}

- Convenient online submission

- Rigorous peer review

- Open access: articles freely available online

- High visibility within the field

- Retaining the copyright to your article

Submit your next manuscript at $\boldsymbol{~ s p r i n g e r o p e n . c o m ~}$ 